\title{
Studies on the clearance of calcium pyrophosphate crystals from facsimile synovium
}

\author{
Y. M. SIN, A. D. SEDGWICK, A. MOORE, AND D. A. WILlOUGHBY \\ From the Department of Experimental Pathology, St Bartholomew's Hospital Medical College, London
}

SUMMARY Calcium pyrophosphate dihydrate crystals have been injected into the air pouch model of facsimile synovium. At various time intervals after crystal injection both infiltrating exudate cells and lining tissue were examined for the presence of crystalline material. The results show that the crystals are initially engulfed by mononuclear cells and to a lesser extent by polymorphonuclear cells in the exudate. Later, crystals became embedded in the lining tissue either within mononuclear cells or as crystal masses confined within granulomas. These studies suggest that the mesenchymal cells (facsimile synovium) lining rat air pouches can clear calcium pyrophosphate dihydrate crystals from the air pouch cavity. It is therefore inferred that crystals found in the lining of human joint synovium may be incorporated therein by a similar mechanism.

It is now generally accepted that crystals can cause inflammation. The presence of these particles in the joint cavity initiates the inflammatory response in 2 diseases: (a) gout, where sodium biurate crystals form with synovial fluid; and $(b)$ pseudogout, where calcium pyrophosphate dihydrate (CPPD) is first precipitated in articular cartilage. ${ }^{1}$ Phelps and McCarty ${ }^{2}$ have suggested that the inflammation seen in synovium following injection of sodium urate crystals occurs only in animals with normal numbers of circulating polymorphonuclear cells. These authors were able to suppress synovitis by the depletion of polymorphonuclear cells with vinblastine. Similarly the inflammation seen after the injection of calcium pyrophosphate dihydrate crystals also involves their phagocytosis by polymorphonuclear cells, thereby activating other mediators of inflammation.

Although the mechanisms involved in the initiation of inflammation by CPPD have been studied extensively, less is known about the fate of crystals once they have been shed into the joint space. ${ }^{3}$ The present study has used the simple air cavity model of facsimile synovium to study the clearance of crystals from connective tissue cavities.

Accepted for publication 28 September 1983.

Correspondence to Professor D. A. Willoughby, Department of Experimental Pathology, St Bartholomew's Hospital Medical College, West Smithfield, London EC1A 7BE.

\section{Materials and methods}

Animals. Male Wistar rats weighing 200-250 g were used throughout the present study. For each experiment not fewer than 6 rats were used for the observation.

Air cavities were produced by the method of Edwards et al. ${ }^{4}$ Rats were injected with $20 \mathrm{ml}$ of air into the subcutaneous tissue of the back. Subsequently $10 \mathrm{ml}$ of air was injected into the same cavity every 3 days to keep the cavity open. Six days after initial air injection the animals were reanaesthetised, and calcium pyrophosphate crystal suspension was introduced with a sterile syringe and needle. Calcium pyrophosphate dihydrate crystals were prepared by a slight modification of the method of Denko and Whitehouse. ${ }^{5}$ A $10 \%$ suspension of crystals was prepared in sterile physiological saline, then ultrasonicated at 15000 cycles per second for 5 minutes. $5 \mathrm{ml}$ of this suspension containing $100 \mathrm{units} / \mathrm{ml}$ penicillin and $100 \mu \mathrm{g} / \mathrm{ml}$ streptomycin was injected into the air pouch. Groups of animals were killed at $2,4,6$, and 24 hours after crystal injection. The total leucocyte number and exudate volume in the cavity were determined. Differential cell counts were also made on smears of exudate cells stained with MayGrunwald giemsa stain.

Histology. 2, 4, 6, 10, 12 hours, and 1, 3, 5, 7, 14 , and 28 days after crystal injection the rats were killed and the pouch linings removed and fixed in $10 \%$ formal saline. Paraffin sections of $6 \mu \mathrm{m}$ thickness 

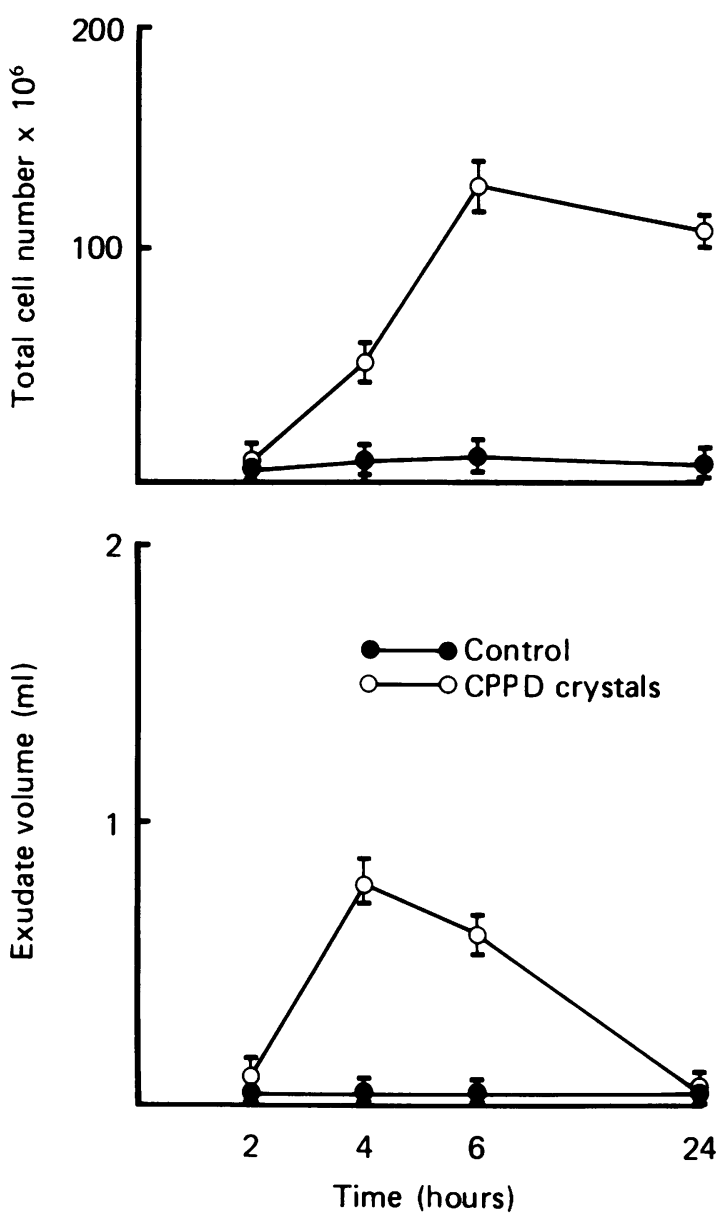

Fig. 1 Total number ofleucocytes and volume of exudate at different time intervals after the injection of calcium pyrophosphate crystals into the pouch.

were cut and stained with toluidine blue, van Gieson, and Martius scarlet blue (MSB) stain.

\section{Results}

The results show the number of leucocytes and the volume of exudate at different time intervals after the injection of calcium pyrophosphate dihydrate crystals into the air pouch (Fig. 1). The maximum number of leucocytes was detected 6 hours after initial injection, whereas the maximum exudate volume occurred at 4 hours. Differential cell counts performed during these early time intervals showed that exudate mononuclear cells were the predominant cell type to contain crystalline material (Fig. 2a). Polymorphonuclear cells were observed to contain crystals less

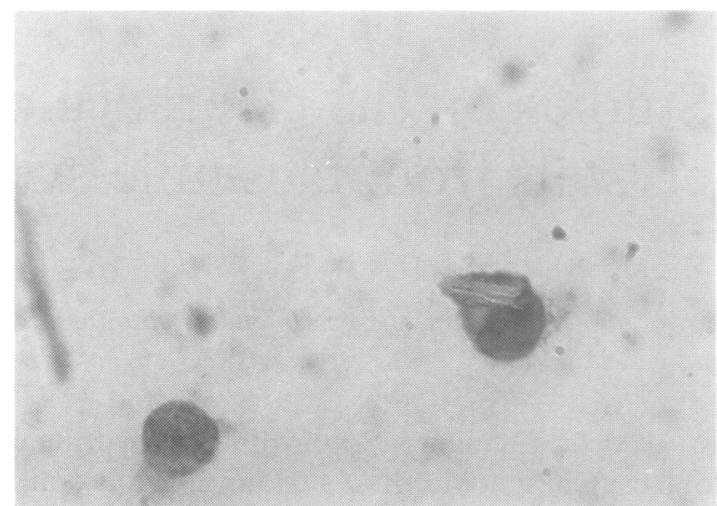

Fig. 2 a Exudate smear from pouch, 2 hours after crystal injection, showing both a polymorphonuclear leucocyte and a mononuclear cell. Note that the mononuclear cell shows a crystal within its cytoplasm. (May-Grunwald Giemsa, $\times 650$, polarised light microscopy).

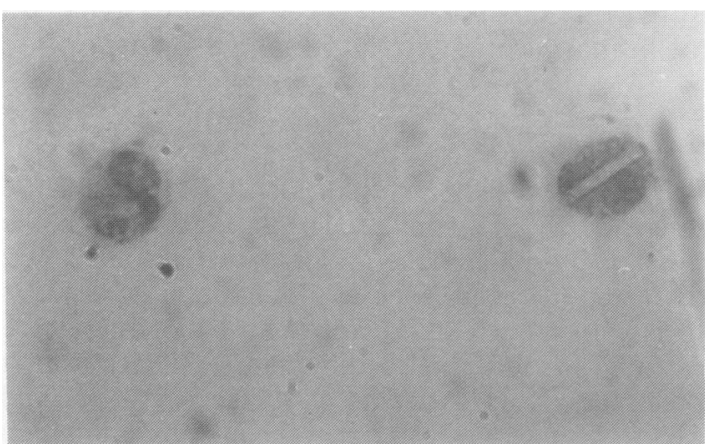

Fig. 2 b Exudate smear from pouch, 2 hours after crystal injection, showing 2 polymorphonuclear cells, one of which contains a crystal in its cytoplasm. (May-Grunwald Giemsa, $\times 650$, polarised light microscopy).

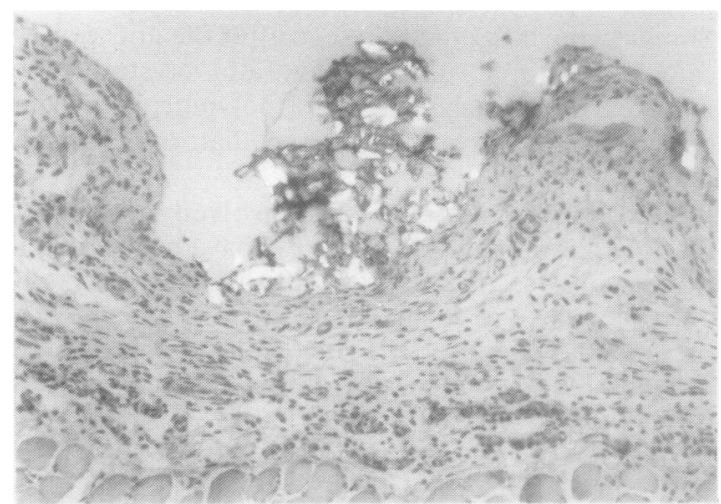

Fig. 2 c A tissue section of pouch wall 2 hours after crystal injection. Note that the crystals are present in the inflamed exudate (seen also at higher magnification in $2(d)$.

(Toluidine blue, $\times 60$, polarised light microscopy). 


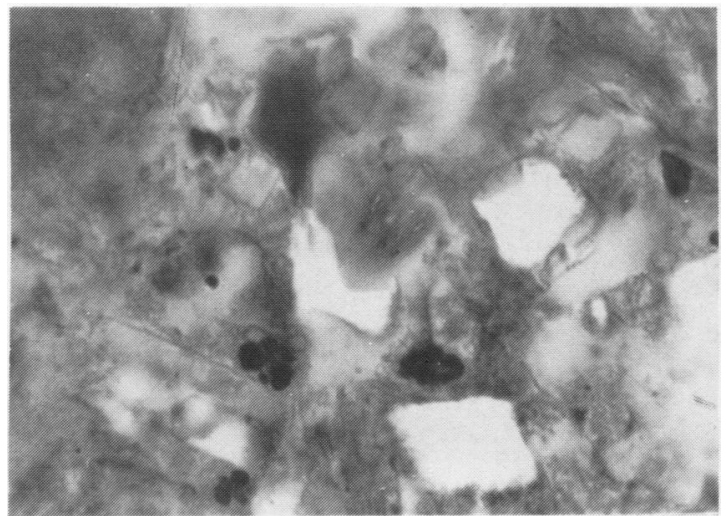

Fig. $2 \mathrm{~d}$ A tissue section of pouch wall 2 hours after crystal injection. Note that the crystals were surrounded by polymorphonuclear leucocytes. (Toluidine blue, $\times 650$, polarised light microscopy).

frequently (Fig. 2b). At 2 hours both exudate cell types were rarely seen to contain crystals. Usually single crystals were found in individual leucocytes, but it was also common to see groups of mononuclear and polymorphonuclear cells surrounding single crystal particles (Figs. 2c, d).

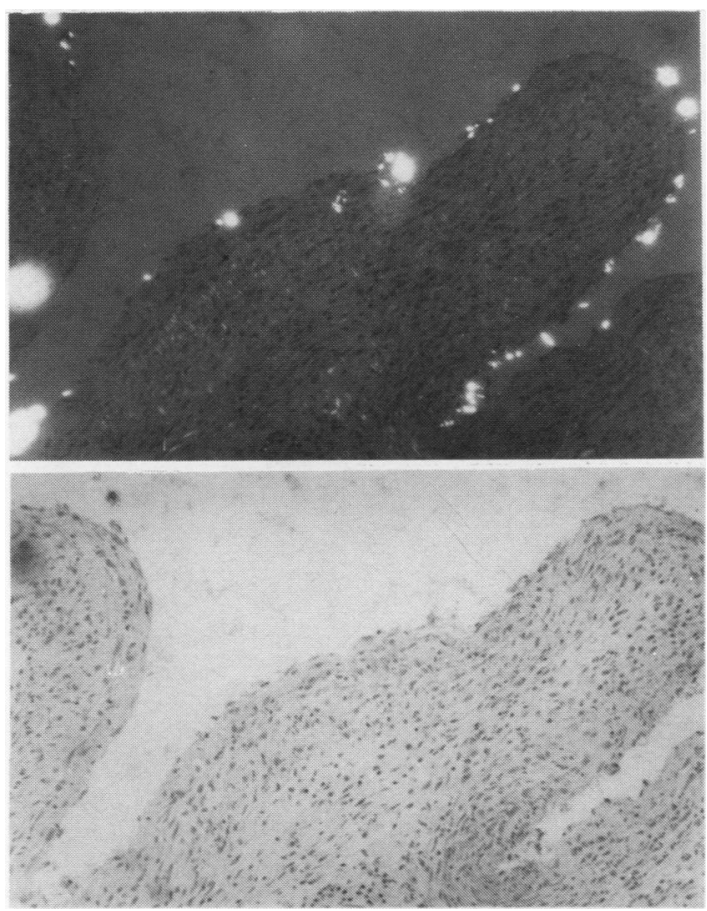

Figs. 3 a and $\mathrm{b}$ A tissue section of pouch wall 4 hours after crystal injection, showing the crystals attached on the surface of the lining tissue. (Toluidine blue, $\times 60,(a)$ polarised light microscopy).
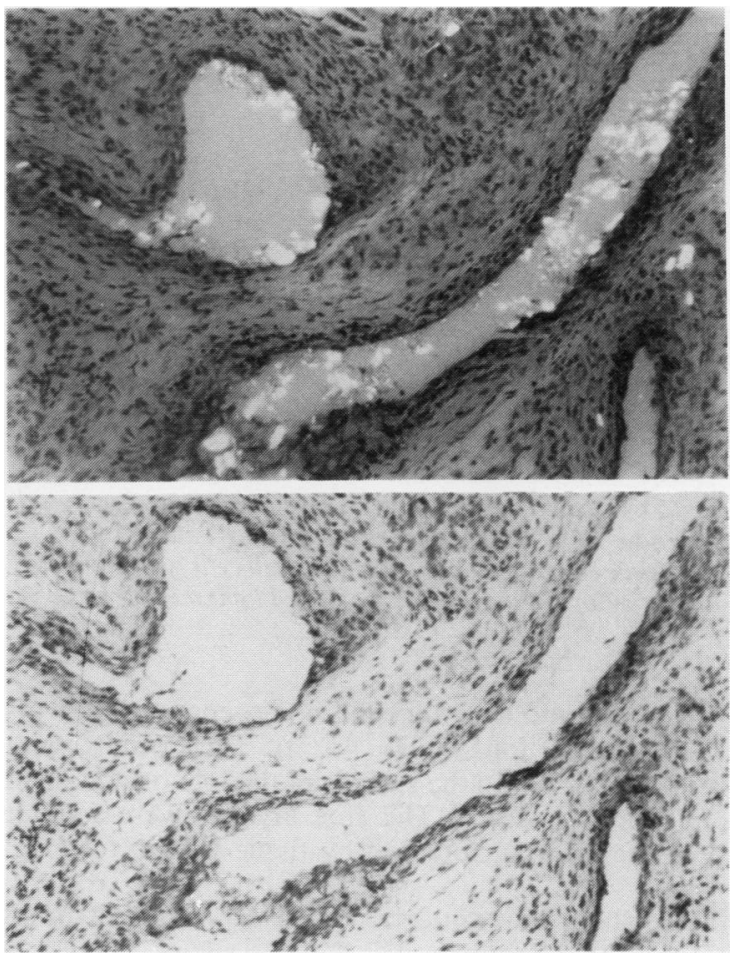

Figs. $4 \mathrm{a}$ and $\mathrm{b}$ A tissue section of pouch wall 6 hours after crystal injection, showing crystals trapped in the spaces between the lining tissue. (Toluidine blue, $\times 60$, (a) polarised light microscopy). Note the crystals were not surrounded by leucocytes.

Two to 4 hours after injection of crystals mononuclear cells containing crystals were observed in the lining tissue. Many crystals were also found attached to the surface of the superficial lining of the pouch (Figs. 3a, b). By 6 hours deep folding of the tissue was

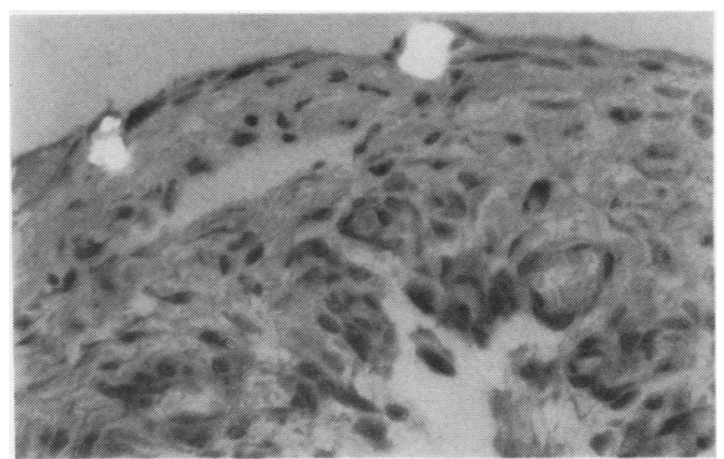

Fig. 5 a Tissue section of pouch wall 10 hours after crystal injection, showing the crystals within the tissue lining. (Toluidine blue, $\times 222$, polarised light microscopy). 


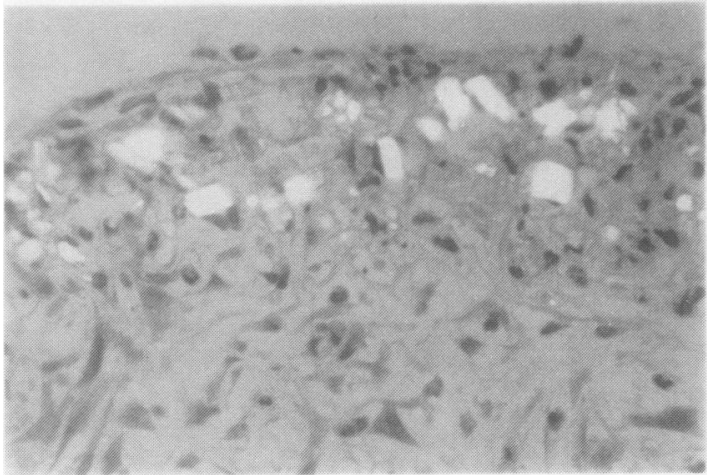

Fig. $5 \mathrm{~b}$ A tissue section of pouch wall 10 hours after crystal injection. Note that the superficial layer of collagen like substance contained only a few scattered cells with elongated nuclei. (Van Gieson, $\times 222$, polarised light microscopy).

observed, and many crystals were seen in the crypts between the folds (Figs. 4a, b). The crystals were either attached to the surface of the fold or remained free in the spaces. The free crystals were neither engulfed nor surrounded by tissue leucocytes (Fig. 4a).

Ten hours after injection many crystals were found within the lining tissue. Fig. 5a shows 2 crystals underneath the cells of the superficial layer of normal appearance. Where large numbers of crystals were deposited on the lining tissue (Fig. 5b) van Gieson staining showed a pink material encapsulating the crystal particles and separating them from the cavity. This layer of collagen-like material appeared incomplete and contained few mononuclear cells. In some pouches this layer was complete, so that an organised lining completely covered the crystals. At this point

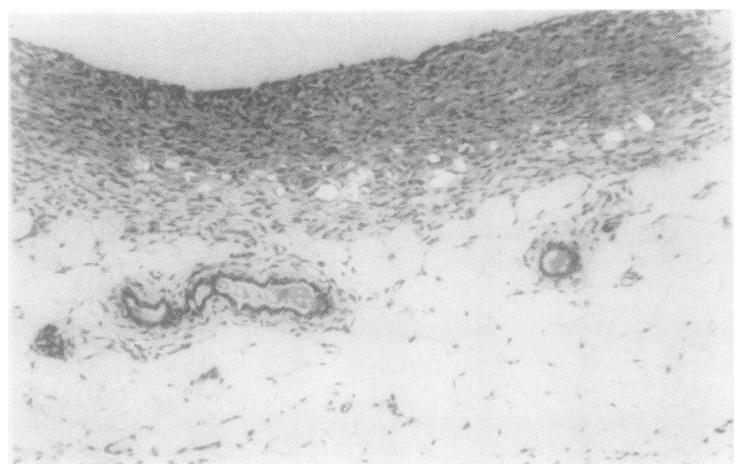

Fig. 6 Pouch tissue section 24 hours after crystal injection. Note that the crystals were embedded deep in the lining tissue. (Toluidine blue, $\times 60$, polarised light microscopy).

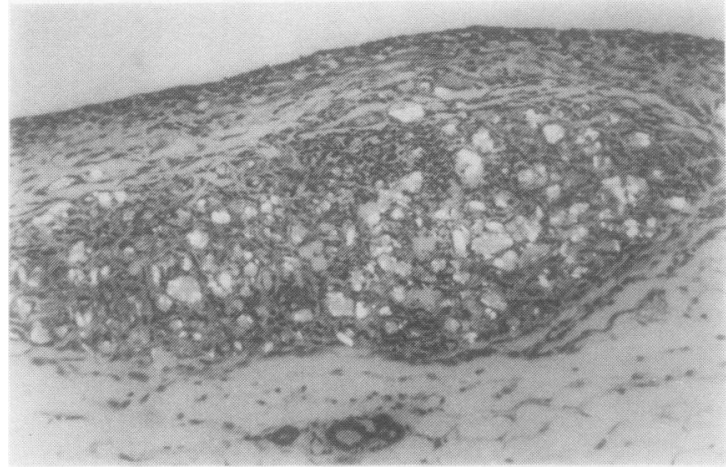

Fig. 7 a A pouch tissue section 3 days after crystal injection, showing a granuloma (seen at higher magnification in 7 (b)) containing crystals and situated deep in the pouch lining. Note that there are many leucocytes among the crystals. (Toluidine blue, $\times 60$, polarised light microscopy).

this tissue resembled the normal appearance of the superficial lining of 6-day-old pouch tissue (Fig. 5a). Crystalline material present in the lining tissue was often found within the mononuclear cells. By 24 hours crystals were found to be deposited in the deeper part of the lining tissue (Fig. 6).

After 3 days various sizes of granulomas containing crystals were seen within the tissue lining. Figs. 7a,b (3-day-old pouch) shows that crystals within the granuloma were present within a dense population of mononuclear and polymorphonuclear cells, the crystals either in individual cells or surrounded by them. Fourteen days after crystal injection, granulomas (Fig. 8a) were found to be surrounded by a few distinct layers of fibroblast-like cells (Fig. 8b). Such granulomas contained fewer leucocytes, and most of the crystals remained free.

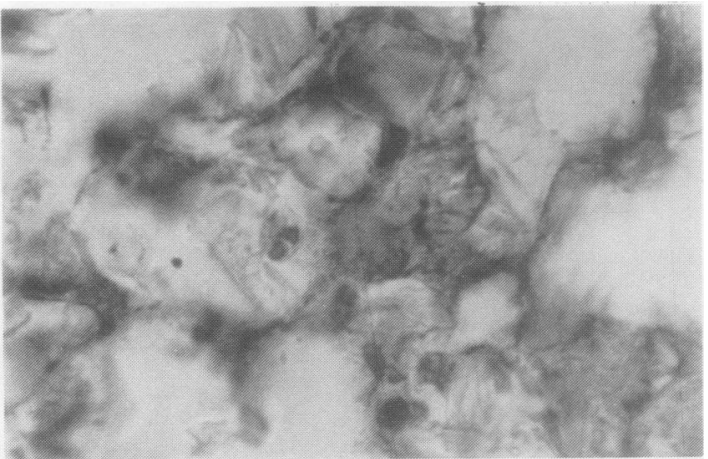

Fig. 7 b Pouch lining tissue section 3 days after crystal injection showing a granuloma containing crystals. Note crystals surrounded by leucocytes. (Toluidine blue, $\times 650$, polarised light microscopy). 


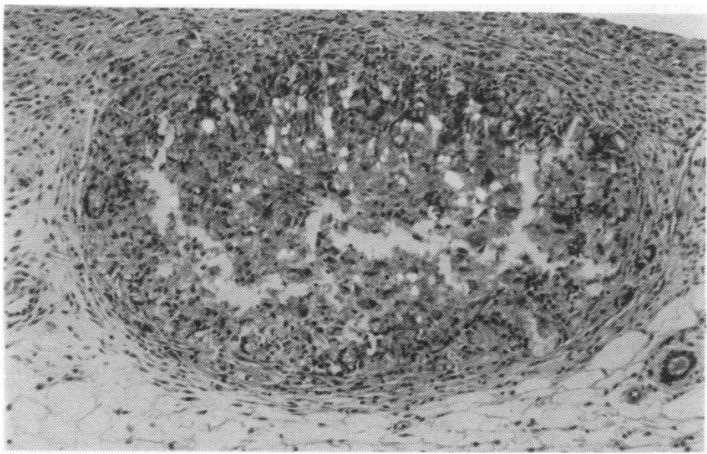

Fig. 8 a Pouch tissue section 14 days after the injection of crystals. Note that the granuloma containing crystals was surrounded by distinct layers of fibroblast-like cells with fewer leucocytes among the crystals. (Toluidine blue, $\times 60$, polarised light microscopy).

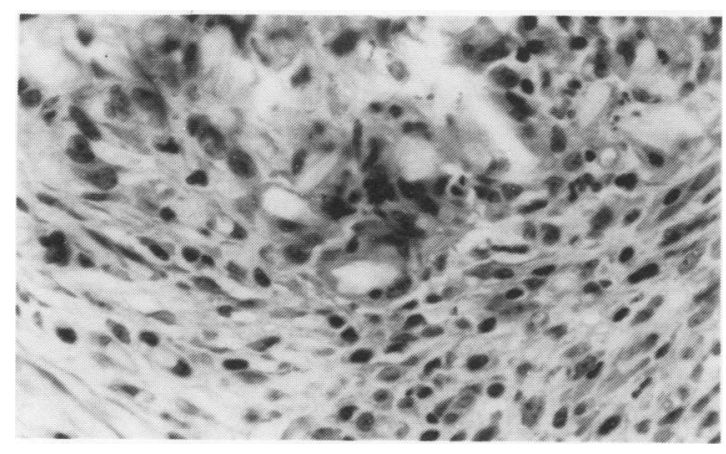

Fig. $8 \mathrm{~b}$ Higher magnification of granuloma-containing crystals. Note the periphery of the granuloma has many mononuclear cells. (Toluidine blue, $\times 222$, polarised light microscopy).

\section{Discussion}

In the present study the 6-day-old air pouch has been used because its lining has the features of synovial tissue. ${ }^{46}$ The study of injury in the joint cavity of rodents is technically very difficult. The air pouch has therefore considerable advantages in that it allows the study of irritants on a tissue which is vascular and has type A and type B synovium-like cells. Acute inflammation in this tissue by CPPD crystals is characterised by polymorphonuclear cell influx and fluid exudation. The maximum leucocyte number in the pouch occurs 6 hours after irritant injection, whereas maximum exudate formation occurs at 4 hours. Denko and Whitehouse ${ }^{5}$ injected CPPD crystals into the footpads of rats and showed that such crystals induce a persistent and severe oedema even
48 hours after crystal injection. The present results show that crystal inflammation in the air pouch is more transient, resolving very quickly. The apparent discrepancy between our results and those of Denko and Whitehouse suggests that inflammation produced with crystals is dependent not only on the crystal type, size, and shape ${ }^{7}$ but also on the nature of the tissue into which it is released or introduced. Since the air pouch has features of synovium, the present studies seem to indicate that synovial tissue is capable of reducing inflammatory reactivity by clearing away crystal particles.

The present studies would suggest that several mechanisms exist for the clearance of crystals: (a) crystals are engulfed by the infiltrating mononuclear phagocytes and to a lesser extent by polymorphonuclear cells; $(b)$ lining surface phagocytic cells engulf crystalline material; $(c)$ crystals are encapsulated by a layer of collagen-like fibres; and $(d)$ crystals are entrapped by the folding of lining tissue.

McCarty et al. ${ }^{8}$ have previously suggested an important role for fixed synovial cells in removing CPPD crystals for the joint space. The present findings confirm the work of these authors that macrophages are important; however, it would seem that encapsulation by collagen-like fibres plays a similarly important role.

It is possible that folding of the tissue results from the rapid decrease in exudate volume, causing tissue to contract. This concept of folding allows speculation as to the mechanisms involved in acute attacks of joint inflammation. Crystals entrapped in the lining tissue may be released in response to oedema caused by trauma or infection inducing tissue secretions. Doyle $^{9}$ has shown that deposits of CPPD in osteoarthritis consist generally of large discrete aggregates of crystals which appear to be the result of local crystal deposition. Such clinical circumstances may lead to the crystals being cleared by encapsulation by collagen-like fibres eventually leading to granuloma formation.

The present study indicates that synovium-like lining tissue plays an important role in the clearance of crystals from cavities. It is therefore likely that in man crystals are cleared in a similar manner. The formation of crystal granulomas may confine the release of biologically active products from the phagocytic cells involved in crystal interaction. Such a mechanism may explain the acute nature of inflammation induced with crystals.

The work was supported by a Commonwealth Academic Staff Fellowship (YMS) and the Arthritis and Rheumatism Council and the Knights Bachelor Fund. 


\section{References}

1 Dieppe P A. Crystal induced inflammation and osteoarthritis. In: Willoughby D A, Giroud J P, Velo G P, eds. Perspectives in infiammation. London: MTP Press, 1977.

2 Phelps P, McCarty D J. Crystal induced inflammation in canine joints. II. Importance of polymorphonuclear leucocytes. J Exp Med 1966; 124: 115-26.

3 McCarty D. Crystals, joints, and consternation. Ann Rheum Dis 1983; 42: 243-53.

4 Edwards J C W, Sedgwick A D, Willoughby D A. The formation of a structure with the features of synovial lining by subcutaneous injection of air: an in vivo tissue culture system.J Pathol $1981 ; 134$ : 147-56.
5 Denko $\mathrm{C} \mathrm{W}$, Whitehouse $\mathrm{M}$ W. Experimental inflammation induced by naturally occurring microcrystalline calcium salts. $J$ Rheumatol 1976; 3: 54-62.

6 Sedgwick A D, Sin Y M, Edwards J C W, Willoughby D A. Increased inflammatory reactivity in newly formed lining tissue. $J$ Pathol in press.

7 Dieppe P A. Osteoarthritis and crystal deposition. In: Panayi G S, ed. Scientific basis of rheumatology. London and Edinburgh: Churchill Livingstone, 1982: 224-41.

8 McCarty D J, Palmer D W, James C. Clearance of calcium pyrophosphate dihydrate (CPPD) crystals in vivo. II. Studies using triclinic crystals doubly labelled with ${ }^{45} \mathrm{Ca}$ and ${ }^{85} \mathrm{Sr}$. Arthritis Rheum 1979; 22: 1122-31.

9 Doyle D V. Tissue calcification and inflammation in osteoarthritis. J Pathol 1982; 136: 199-216. 